\title{
Associations of Race, Insurance, and Zip Code- Level Income with Nonadherence Diagnoses in Primary and Specialty Diabetes Care
}

\author{
Sourik Beltrán, MD, MBE, Daniel J. Arenas, PhD, Itzel J. López-Hinojosa, BA, \\ Elizabeth L. Tung, MD, MS, and Peter F. Cronbolm, MD, MSCE
}

Introduction: Evidence suggests that clinicians may view or label patients as nonadherent in a biased manner. Therefore, we performed a retrospective cohort analysis exploring associations between patient demographics and zip code-level income with the International Classification of Diseases, Tenth Version (ICD10) diagnoses for nonadherence among type 2 diabetes mellitus (T2DM) patients, comparing primary and specialty care settings. Providers in the primary care group included internal medicine and family medicine physicians. In the specialty care group, providers included endocrinologists and diabetologists only.

Methods: Participants were identified from 5 primary care and 4 endocrinology sites in the University of Pennsylvania Health System between January 1, 2015, and January 1, 2019.

Demographics, hemoglobin A1c (HbA1c), and ICD-10 codes for T2DM and nonadherence were extracted from the electronic health record and analyzed in October 2019. Log-binomial regression models were used to estimate patients' risk of nonadherence labeling by race, insurance, and zip codelevel median household income, controlling for patient characteristics and HbA1c as a proxy for diabetes self-management. Results were compared between primary and specialty care sites.

Results: A total of 6072 patients aged 18-70 years were included in this study. Black race, Medicare, and Medicaid were associated with increased nonadherence labeling while controlling for patient characteristics ([ARR $=2.48,95 \%$ CI: 2.01, 3.04], [ARR $=1.82,95 \%$ CI: 1.50, 2.18],

[ARR = 1.61, 95\% CI: 1.32, 1.93], respectively). The results remained significant on adjustment with zip code-level income and showed no differences between primary and specialty sites. Lower-income zip codes showed a significant association with increased rates of nonadherence labeling.

Conclusions: Black race, non-private insurance, and lower-income zip codes were associated with disproportionately high rates of nonadherence labeling in both primary and specialty management of T2DM, possibly suggestive of racial or class bias. ( J Am Board Fam Med 2021;34:891-897.)

Keywords: HbA1c, Type 2 Diabetes Mellitus, Patient Compliance, Poverty, Primary Health Care, Retrospective Studies, Nonadherence, Patient Labeling, Bias, Race, Socioeconomic Status

\section{Introduction}

Nonadherence is a widely used term referring to patients' low engagement with lifestyle modifications,

This article was externally peer reviewed.

Submitted 16 December 2020; revised 19 April 2021; accepted 20 April 2021.

From the Department of Medicine, Massachusetts General Hospital, Boston, MA (SB); Perelman School of Medicine, University of Pennsylvania Philadelphia, PA (DJA); Department of Medical Ethics and Health Policy, University of Pennsylvania (SB); Pritzker School of Medicine, University of Chicago, Chicago, IL (IJLH); Department of Medicine, Section of General Internal Medicine, University of Chicago (ELT); Chicago Center for Diabetes Translation Research, University of Chicago (ELT); Department of Family Medicine and Community Health, University of Pennsylvania (PFC); Center for Public Health Initiatives, University of medication use, or outpatient follow-up. ${ }^{1-4}$ Low engagement can be due to many reasons, including structural issues like transportation and financial difficulty. ${ }^{5-7}$ As a result, the term "nonadherence" has received criticism for potentially obscuring intervenable barriers to care while placing blame on patients for their disease states. ${ }^{8}$ Moreover, the term has been

Pennsylvania (PFC); Leonard Davis Institute of Health Economics, University of Pennsylvania (PFC).

Funding: None.

Conflict of interest: None.

Corresponding author: Sourik Beltrán, MD, MBE, 55 Fruit St., Boston, MA 02114, Phone: 248-444-5143, Fax: 215-898-0833 (E-mail: sbeltran@partners.org). 
associated with paternalistic attitudes and clinician bias which can negatively impact patient care. ${ }^{9-11}$

Prior studies have demonstrated that patients' race, sex, and socioeconomic status (SES) can skew providers' perceptions of health behaviors and overall cooperativeness. ${ }^{12-17}$ Given that nonadherence terms may themselves carry stigma, their use in patients' electronic health records (EHRs) may be problematic, as they may not only arise from but also worsen provider bias.

In a recent study of 3768 adults, we showed that Black and non-privately insured patients with type 2 diabetes mellitus (T2DM) were disproportionately labeled "nonadherent" compared with White, privately insured patients. These findings persisted even after controlling for HbA1c as a proxy for diabetes self-management. ${ }^{18}$ Nevertheless, no study to date has explored the use of nonadherence labels in different care settings or how measures of SES may influence nonadherence labeling. Therefore, we conducted a cross-sectional retrospective cohort analysis to examine sociodemographic characteristics associated with nonadherence diagnoses and explore potential differences in their application between primary and specialty care sites. We also examined zip code-level median household income as a proxy for neighborhood disadvantage.

\section{Methods}

Participant data were retrospectively retrieved from EHRs of patients with T2DM who received care at 1 of 4 primary care or 5 endocrinology sites in the University of Pennsylvania Health System between January 1, 2015, and January 1, 2019. The study was approved by the University of Pennsylvania Institutional Review Board in June 2019. Extracted patient characteristics included age, gender, BMI, HbA1c, self-identified race and ethnicity, insurance, healthcare site, and ICD-10 nonadherence diagnoses (ICDZ91.1: "patient's noncompliance with medical treatment and regimen" or ICDZ53.2: "procedure and treatment not conducted because of patient's decision" [S1]).

Patient zip codes were also retrieved from the EHR and paired with publicly available data on median household income of Philadelphia zip codes. ${ }^{19}$ The effect of categorical variables on the probability of having at least 1 nonadherence diagnosis was calculated using risk ratios (RR). Logistic regression models were used to adjust for age, HbA1c, body mass index (BMI), sex, race, ethnicity, and insurance. ${ }^{20}$ $\beta$ eta $(\beta)$ coefficients from a generalized linear model were used for numeric variables. Significance was estimated using 95\% confidence intervals (95\% CI). Multiple hypothesis corrections were not applied to prevent low statistical power. ${ }^{21}$ Document S1 offers additional methodological details.

\section{Results}

The final cohort included 6072 patients, $12.5 \%$ labeled nonadherent. Cohort characteristics are shown in Table 1 with participant selection details in document $\mathrm{S} 1$.

We found positive associations between Black race, Medicaid, and Medicare with nonadherence labeling after adjusting for covariates (adjusted RR $[\mathrm{ARR}]=2.48,95 \%$ CI: 2.01, 3.04; $\mathrm{ARR}=1.82,95 \%$ CI: $1.50,2.18$; $\mathrm{ARR}=1.61,95 \% \mathrm{CI}: 1.32,1.93$; respectively, Table 2). Patients with higher HbA1c levels showed significantly increased risk of nonadherence labeling $(\beta$ adj $=0.11[0.08,0.15]$, Table 2$)$. Notably, when analyzing patients with $\mathrm{HbA1c}<7 \%$, Black and non-privately insured patients still showed a higher risk of nonadherence labeling (Table 2).

For our primary hypothesis, we found that patients at specialty sites were less likely than those in primary care sites to be labeled nonadherent before covariate adjustment $(\mathrm{RR}=0.79,95 \% \mathrm{CI}$ : $0.69,0.91)$. However, the adjusted RR showed no association $(\mathrm{ARR}=1.0,95 \% \mathrm{CI}$ : 0.85, 1.16; Table 2). Subgroup analysis showed the risk of nonadherence labeling for Blacks relative to Whites was similar between primary and specialty sites $(\mathrm{ARR}=2.56$, 95\% CI: 1.66, 3.97; $\mathrm{ARR}=2.45$, 95\% CI: 1.90 , 3.16; respectively, Table 2).

Next, we explored how markers of SES could impact nonadherence labeling. Using data for 47 Philadelphia zip codes corresponding to 3591 participants, we found lower-income zip codes were significantly associated with increased nonadherence labeling after covariate adjustment $(\beta \mathrm{adj}=$ -0.027 [-0.041, -0.014], Table 3). The association persisted in the HbAlc $<7 \%$ stratum and did not differ across primary or specialty sites.

Finally, as zip code-level income was found to be significantly associated with nonadherence diagnoses, we explored how adjusting for this variable would influence nonadherence labeling for race and insurance. We found that Black patients remained at significantly elevated risk of nonadherence labeling after 
Table 1. Sample Characteristics with Crude Risk Ratios (RRs) for Nonadherence Labeling

\begin{tabular}{|c|c|c|c|c|c|}
\hline Variable & $\begin{array}{c}\text { Overall } \\
(\mathrm{N}=6072) \\
\text { Median [IQR] }\end{array}$ & $\begin{array}{c}\text { Labeled } \\
(\mathrm{N}=759) \\
\text { Median [IQR] }\end{array}$ & $\begin{array}{c}\text { Non-Labeled } \\
(\mathrm{N}=5313) \\
\text { Median [IQR] }\end{array}$ & $\boldsymbol{\beta}[95 \% \mathrm{CI}]$ & $P$ value \\
\hline Age, years & $57[49,63]$ & $56[48,62]$ & $57[49,63]$ & $-0.0013[-0.0021,-0.0005]$ & .001 \\
\hline HbAlc, \% & $7.5[6.6,9.1]$ & $8.3[6.8,10.0]$ & $7.5[6.5,8.9]$ & $0.019[0.015,0.023]$ & $<.001$ \\
\hline BMI, $\mathrm{kg} / \mathrm{m}^{2}$ & $\begin{array}{l}32.3[27.8,37.9] \\
\mathrm{n}(\%)\end{array}$ & $\begin{array}{l}32.5[27.5,38.9] \\
\mathrm{n}(\%)\end{array}$ & $\begin{array}{l}32.3[27.8,37.8] \\
\mathrm{n}(\%)\end{array}$ & $\begin{array}{l}0.0007[-0.0003,0.002] \\
\operatorname{RR}[95 \% \mathrm{CI}]\end{array}$ & $\begin{array}{l}.53 \\
P \text { value }\end{array}$ \\
\hline \multicolumn{6}{|l|}{ Biological sex } \\
\hline Male & $2909(47.9)$ & $333(43.8)$ & $2576(48.4)$ & Ref & Ref \\
\hline Female & $3163(52.1)$ & $426(56.1)$ & $2737(51.5)$ & $1.17[1.03,1.35]$ & .017 \\
\hline \multicolumn{6}{|l|}{ Race } \\
\hline White & $1941(31.9)$ & $106(14.0)$ & $1835(34.5)$ & Ref & Ref \\
\hline Black & $3753(61.8)$ & $623(82.1)$ & $3130(58.9)$ & $3.04[2.49,3.71]$ & $<.001$ \\
\hline Asian & $229(3.8)$ & $10(1.3)$ & $219(4.1)$ & $0.80[0.42,1.51]$ & .49 \\
\hline \multicolumn{6}{|l|}{ Ethnicity } \\
\hline Non-Hispanic & $5906(97.3)$ & $739(97.4)$ & $5167(97.3)$ & Ref & Ref \\
\hline Hispanic & $166(2.7)$ & $20(2.6)$ & $146(2.7)$ & $0.96[0.63,1.46]$ & .85 \\
\hline \multicolumn{6}{|l|}{ Insurance payor } \\
\hline Private & $3536(58.2)$ & $335(44.1)$ & $3201(60.2)$ & Ref & Ref \\
\hline Medicaid & $1082(17.8)$ & $222(29.2)$ & $860(16.2)$ & $2.17[1.85,2.52]$ & $<.001$ \\
\hline Medicare & $1454(23.9)$ & $202(26.6)$ & $1252(23.6)$ & $1.44[1.23,1.70]$ & $<.001$ \\
\hline \multicolumn{6}{|l|}{ Care site } \\
\hline Primary care & $2657(43.8)$ & $376(49.5)$ & $2281(42.9)$ & Ref & Ref \\
\hline Specialty care & $3415(56.2)$ & $383(50.5)$ & $3032(57.1)$ & $0.79[0.69,0.91]$ & $<.001$ \\
\hline
\end{tabular}

BMI, body mass index; CI, confidence interval; HbA1c, hemoglobin A1C; IQR, interquartile range.

adjusting for zip code (ARR $=1.70,95 \% \mathrm{CI}$ : 1.28 , 2.30; Table 3). Medicare and Medicaid also showed significantly increased risk $(\mathrm{ARR}=1.71,95 \% \mathrm{CI}$ : 1.39, 2.07; ARR $=1.61,95 \%$ CI: 1.29, 1.99; Table 3) compared with private insurance. The results persisted in subgroup analyses for HbAlc above and below $7 \%$ and in primary and specialty sites.

\section{Conclusions}

In a clinical sample of 6072 T2DM patients, we observed a higher risk of nonadherence labeling associated with the Black race, non-private insurance, and lower-income zip code. This study additionally presents novel findings on the use of nonadherence diagnoses in primary and specialty care settings.

We demonstrated no difference between primary or specialty care sites in the associations of Black race, non-private insurance, and lowerincome zip code with nonadherence diagnoses even when controlling for HbA1c. Considering prior studies that showed patient demographics could influence clinicians' perceptions of self-management behavior, ${ }^{12-16}$ our findings may signify similar bias leading to uneven utilization of nonadherence labels in T2DM care. However, additional studies would be needed to characterize the precise causes of uneven nonadherence labeling.

We hypothesized that nonadherence diagnoses would be lower in primary care sites due to the depth and continuity of primary care relationships possibly mitigating bias. In addition, we reasoned that endocrinology referrals are reserved for patients with more complex care needs, which could increase patient difficulty with treatment recommendations. However, if nonadherence labeling is indeed related to bias, the observation of similar results between primary and specialty care sites may reflect comparable levels of bias across medical specialties, a finding consistent with prior research. ${ }^{22,23}$

Another novel finding was that lower-income zip codes were associated with increased nonadherence labeling even when correcting for covariates. This finding may support the hypothesis that bias may lead providers to perceive T2DM patients of lower SES as less engaged in their care compared with higher-income patients. Alternatively, it could indicate a lack of provider knowledge regarding the healthcare challenges lower-income patients face. 


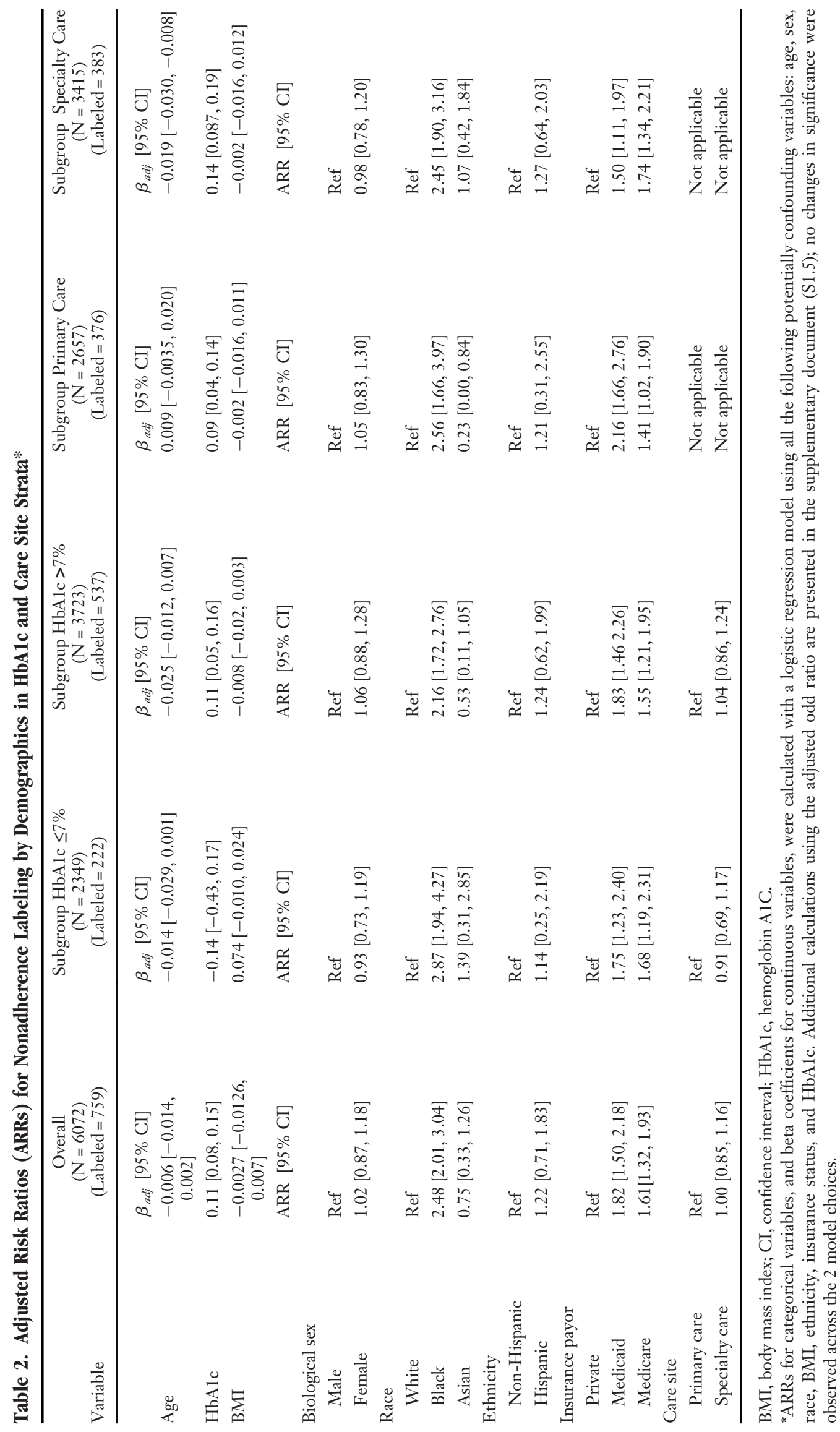




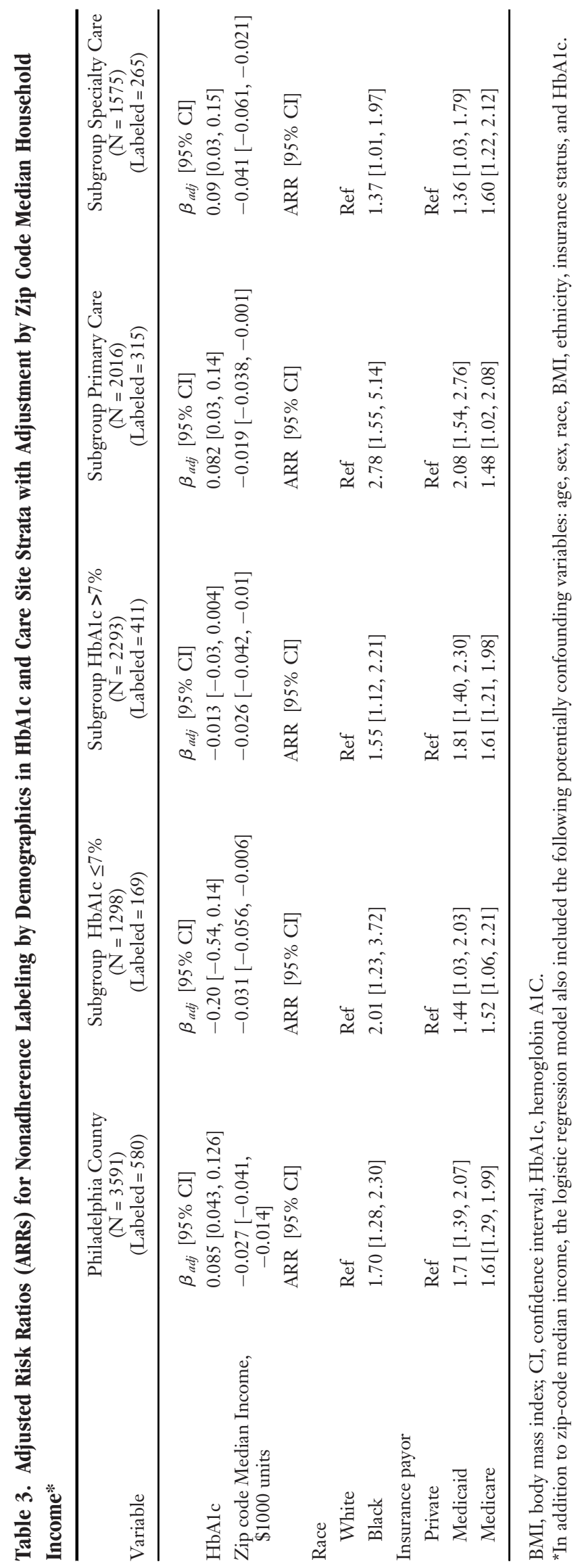


Given the likely multifactorial nature of nonadherence labeling and the limitations of low-granularity data like zip code, ${ }^{24}$ further research is needed to explore relationships between SES, health behaviors, and provider perceptions.

Our results showed that Black patients were at significantly higher risk of being labeled nonadherent after adjustment for covariates, including HbA1c and income. We observed similarly disproportionate nonadherence labeling among Black patients with $\mathrm{HbA1c}<7 \%$. These findings raise concern that racial bias may influence the use of nonadherence diagnoses in the EHR. Nevertheless, regardless of the reasons behind nonadherence labeling, our findings are still alarming given that such terms carry negative connotations, which may subsequently lead to worse care. ${ }^{8-11}$

This study has several limitations. As a cross-sectional study, we cannot investigate temporal trends in $\mathrm{HbA1c}$, BMI, or insurance nor their longitudinal effect on nonadherence labeling. It is worth noting that HbA1c is most indicative of patients' disease states and thus may not reliably represent engagement in care. Further, our cross-sectional methodology makes it difficult to discern whether patients' nonadherence diagnoses were specifically related to T2DM and not another comorbidity. Finally, due to limited data on provider demographics, we could not explore if our findings differed by provider background.

In this study, we used publicly available data on zip code-level median household income as a surrogate for neighborhood disadvantage. However, as many patients had addresses outside of Philadelphia, we were unable to conduct this analysis on the full sample. Further, zip code-level data provide insufficient granularity to draw reliable conclusions regarding patient SES, ${ }^{24}$ although associations may inform future hypotheses.

Next, we were unable to assess the role of other well-known determinants of diabetes self-management like education and health literacy. The precise behavior to which nonadherence diagnoses may be referring (eg, medication underuse) and clinicians' rationale in the use of those diagnoses remain unclear. Future studies should consider qualitative methodologies to directly explore providers' attitudes regarding T2DM self-management in highrisk populations.

Despite these limitations, our study suggests that nonadherence diagnoses may be unevenly utilized in the EHR for Black and lower-income patients. Our results may cast doubt on the validity of nonadherence labels as they may be more indicative of providers' biases or structural healthcare barriers than patient behavior alone. Our findings may suggest a need for clinicians to exercise greater skepticism when encountering prior documentation of "nonadherence" in patients' health records. We recommend that providers rely less on such labels and instead work to identify specific barriers to patient engagement.

Given that Black and economically disenfranchised patients face disproportionate challenges within US healthcare systems, ${ }^{25,26}$ the identification of sources of bias is critical to mitigating health disparities. Our study may indicate a need to explore further how nonadherence and related language may result from and perpetuate bias in healthcare.

To see this article online, please go to: http://jabfm.org/content/ 34/5/891.full.

\section{References}

1. Kardas P, Lewek P, Matyjaszczyk M. Determinants of patient adherence: a review of systematic reviews. Front Pharmacol 2013;4:1-16.

2. Munger MA, Van Tassell BW, LaFleur J. Medication nonadherence: an unrecognized cardiovascular risk factor. Med Gen Med 2007;9:58.

3. Parajuli J, Saleh F, Thapa N, Ali L. Factors associated with nonadherence to diet and physical activity among Nepalese type 2 diabetes patients; a cross sectional study. BMC Res Notes 2014;7:758.

4. Tola HH, Tol A, Shojaeizadeh D, Garmaroudi G. Tuberculosis treatment nonadherence and lost to follow up among TB patients with or without HIV in developing countries: a systematic review. Iran J Public Health 2015;44:1-11.

5. Hugtenburg JG, Timmers L, Elders PJ, Vervloet $M$, van Dijk L. Definitions, variants, and causes of nonadherence with medication: a challenge for tailored interventions. Patient Prefer Adherence 2013;7:675-82.

6. Syed ST, Gerber BS, Sharp LK. Traveling towards disease: transportation barriers to health care access. J Community Health 2013;38:976-93.

7. Osborn CY, Kripalani S, Goggins KM, Wallston KA. Financial strain is associated with medication nonadherence and worse self-rated health among cardiovascular patients. J Health Care Poor Underserved 2017;28:499-513.

8. Brunton S. I have never liked the term "compliance". Clin Diabetes 2017;35:76-7.

9. Wens J, Vermeire E, Royen PV, Sabbe B, Denekens J. GPs' perspectives of type 2 diabetes patients' adherence to treatment: a qualitative analysis of barriers and solutions. BMC Fam Pract 2005;6:20. 
10. Roberts KJ, Volberding P. Adherence communication: a qualitative analysis of physician-patient dialogue. AIDS 1999;13:1771-8.

11. Wong MD, Cunningham WE, Shapiro MF, HCSUS Consortium, , et al Disparities in HIV treatment and physician attitudes about delaying protease inhibitors for nonadherent patients. J Gen Intern Med 2004; 19:366-74.

12. Dehon E, Weiss N, Jones J, Faulconer W, Hinton E, Sterling S. A systematic review of the impact of physician implicit racial bias on clinical decision making. Acad Emerg Med 2017;24:895-904.

13. Haider AH, Schneider EB, Sriram N, et al. Unconscious race and social class bias among acute care surgical clinicians and clinical treatment decisions. JAMA Surg 2015;150:457-64.

14. Huizinga MM, Bleich SN, Beach MC, Clark JM, Cooper LA. Disparity in physician perception of patients' adherence to medications by obesity status. Obesity (Silver Spring) 2010;18:1932-7.

15. Bogart LM, Catz SL, Kelly JA, Benotsch EG. Physician decision making for HIV factors influencing physicians' judgments of adherence and treatment decisions for patients with HIV disease. Med Decis Making 2001;21:28-36.

16. Lutfey KE, Ketcham JD. Patient and provider assessments of adherence and the sources of disparities: evidence from diabetes care. Health Serv Res 2005;40:1803-17.

17. Green AR, Carney DR, Pallin DJ, et al. Implicit bias among physicians and its prediction of thrombolysis decisions for black and white patients. J Gen Intern Med 2007;22:1231-8.
18. Beltran S, Lett L, Cronholm PF. Nonadherence labeling in primary care: bias by race and insurance type for adults with type 2 diabetes. Am J Prev Med 2019;57(5):652-658.

19. Zip Atlas. Median Household Income in Philadelphia, PA by Zip Code. Available from: http://zipatlas.com/ us/pa/philadelphia/zip-code-comparison/medianhousehold-income.htm. Accessed October 1, 2019.

20. Lee Y. Adjusted Relative Risk from Logistic Regression. Available from: https://github.com/youjin1207/ logisticRR. Accessed October 1, 2019.

21. Brookes ST, Whitely E, Egger M, et al. Subgroup analyses in randomized trials: risks of subgroup-specific analyses; power and sample size for the interaction test. J Clin Epidemiol 2004;57:229-36.

22. Johnson TJ, Winger DG, Hickey RW, et al. Comparison of physician implicit racial bias toward adults versus children. Acad Pediatr 2017; 17:120-6.

23. Kikano GE, Schiaffino MA, Zyzanski SJ. Medical decision making and perceived socioeconomic class. Arch Fam Med 1996;5:267-70.

24. Grubesic TH, Matisziw TC. On the use of ZIP codes and ZIP code tabulation areas (ZCTAs) for the spatial analysis of epidemiological data. Int $\mathrm{J}$ Health Geogr 2006;5:58.

25. Orsi JM, Margellos-Anast H, Whitman S. BlackWhite health disparities in the United States and Chicago: a 15-year progress analysis. Am J Public Health 2010;100:349-56.

26. Braveman PA, Kumanyika S, Fielding J, et al. Health disparities and health equity: the issue is justice. Am J Public Health 2011;101:S149-55. 


\section{Appendix. Additional Methodology Information}

Nonadherence labeling was assigned to patients with either a diagnosis of ICDZ91.1 ("patient's noncompliance with medical treatment and regimen") or ICDZ53.2 code ("procedure and treatment not carried out because of patient's decision for other and unspecified reasons") present in their medical history or problem list. These ICD-10 diagnoses denote patient nonadherence in patients' electronic health records, which are thereby viewable to any provider within the University of Pennsylvania Health System (not just the clinician who made the diagnosis). Further, these diagnoses do not provide specification regarding the exact treatment in question or the reason the diagnosis was given. Race was characterized as Black, White, or Asian. Other racial categories were excluded due to small sample sizes (Appendix Table 1). Ethnicity was limited to Hispanic or non-Hispanic. Last known insurance status was retrieved and categorized as either private, Medicare, or Medicaid.

Patient characteristics were described using the medians and upper and lower quarter ranges. Unadjusted associations between nonadherence labeling and patient characteristics were calculated using crude risk ratios (RRs). Adjusted risk ratios (ARRs) were calculated with log-binomial regression models using all of the following potentially confounding variables: age, sex, race, last recorded body mass index (BMI), ethnicity, insurance status, and average hemoglobin A1c (HbA1c). The 95\% CI of the ARRs was calculated using a bootstrap method of more than 1000 simulations. ${ }^{P}$ RRs were preferred over odd ratios as the prevalence of nonadherence labeling was not low. Nonetheless, to test the effect of model choice on the significance results, adjusted odd ratios (AORs) were also calculated using the standard generalized linear models (Appendix Table 2). ${ }^{2}$ Either the AOR or ARR choice did not change any of the significant results.

The association between numerical variables (ie, zip code-level income) and the prevalence of nonadherence labeling with each zip code's median income was investigated using generalized linear models accounting for the variables previously listed.
Statistical significance was assessed by overlap of $95 \%$ confidence intervals (eg, overlap of ARR with one, and beta coefficients with zero). Lastly, subgroup analyses examined how effect sizes changed in different stratifications (ie, nonadherence labeling in HbA1c strata above and below 7\%; see Appendix Table 3). All statistical analysis was carried out using $\mathrm{R}$ packages.

\section{Participant Selection}

A total of 7232 patients were initially identified with T2DM, ages 18 to 70 , and who received care at any of the four primary care sites or the five specialty care sites included in this study (see Appendix Tables 4 and 5). We only analyzed data pertaining to patients with self-identified races of Black, White, or Asian. Other races, or nonspecified, were excluded due to sizes smaller than 100. (The unadjusted prevalences of nonadherence of all of the groups are presented in Appendix Table 1). In total, 691 participants were excluded from analysis because of missing information (Appendix Figure 1). An additional 45 individuals were excluded due to $\mathrm{BMI}<18$ and 6 due to BMIs greater than 102 (Appendix Figure 1). The final cohort with values available for all variables of interest consisted of 6072 patients for which $12.5 \%$ [95\% CI, 11.7-13.4] were labeled nonadherent by one or both nonadherence diagnoses, ICD10 Z91.1 ( $\mathrm{n}=429)$ or Z53.2 ( $\mathrm{n}=391)$.

\section{Additional Results}

BMI, age, gender, Asian race, and Hispanic ethnicity showed no association with nonadherence labeling after adjustment.

\section{References}

1. Lee Y [Internet]. Adjusted Relative Risk from Logistic Regression. 2020. Available from: https:// github.com/youjin1207/logisticRR.

2. Bates D, Mächler M, Bolker B, Walker S. Fitting linear mixed-effects models using lme4. J Statistical Software 2015;67:1-48. doi: 10.18637/jss.v067.i01. 
Appendix Figure 1. Flowchart demonstrating subject counts for inclusion and exclusion from study.

Initial Cohort

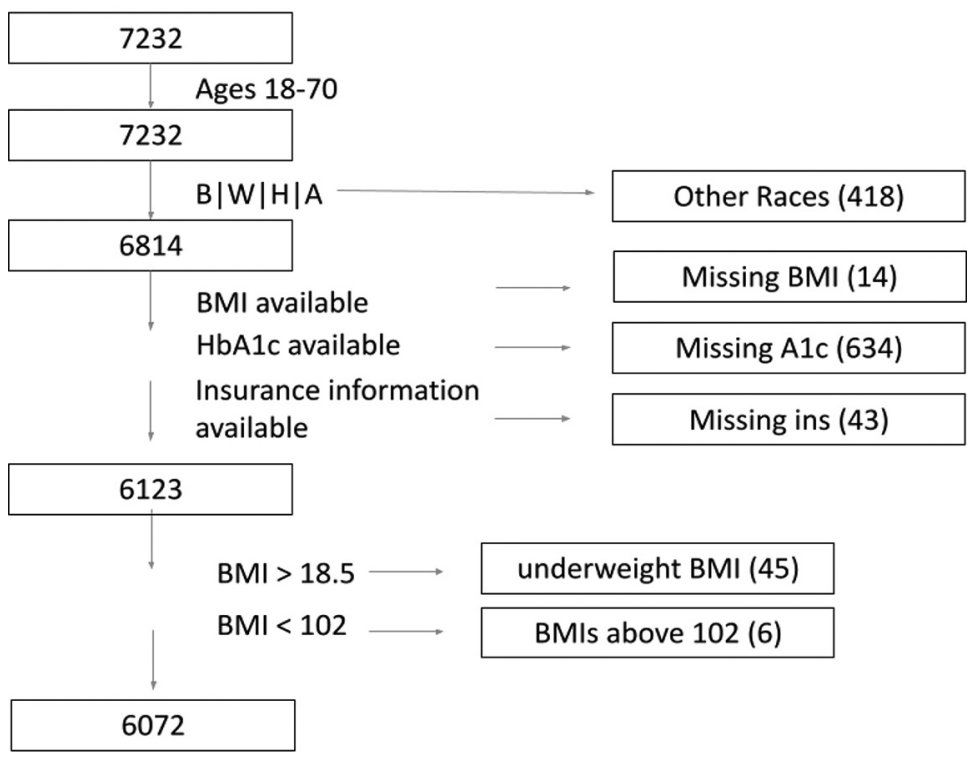

Final Cohort

Appendix Table 1. Unadjusted prevalences of nonadherence labeling among all racial categories

\begin{tabular}{lcccc}
\hline & Total & Not Labeled & Labeled & Prevalence \\
\hline Overall & 7232 & 6405 & 827 & 0.114 \\
Black & 4152 & 3487 & 665 & 0.160 \\
White & 2224 & 2111 & 113 & 0.051 \\
Asian & 260 & 250 & 10 & 0.038 \\
Other & 222 & 211 & 11 & 0.050 \\
Hispanic /Latino_White & 116 & 106 & 10 & 0.086 \\
Hispanic /Latino-Black & 62 & 51 & 11 & 0.177 \\
East Indian & 71 & 67 & 4 & 0.056 \\
American Indian & 19 & 19 & 0 & 0.000 \\
Patient declined & 11 & 11 & 0 & 0.000 \\
Pacific Islander & 4 & 4 & 3 & 0.000 \\
Unknown race & 91 & 88 & 0.033 \\
\hline
\end{tabular}




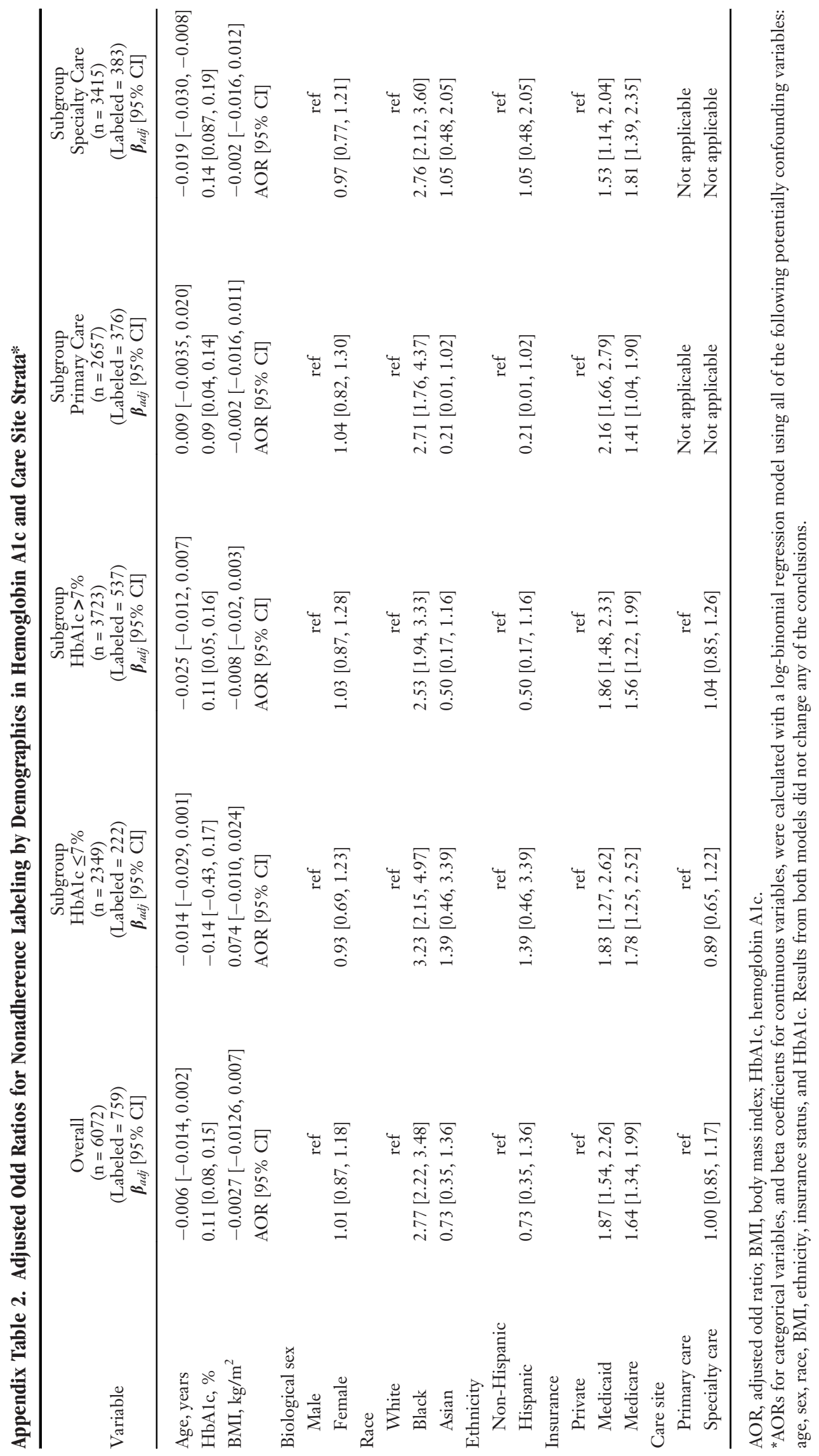

E3 JABFM September-October 2021 Vol. 34 No. 5 
Appendix Table 3. Subgroup Analyses Exploring Different Hemoglobin A1c Cutoffs: 7\% and 9\%

\begin{tabular}{|c|c|c|c|c|c|}
\hline & $\begin{array}{c}\text { Overall } \\
(\mathrm{n}=6072) \\
(\text { Labeled = 759) } \\
\text { ARR }[95 \% \mathrm{CI}]\end{array}$ & $\begin{array}{c}\text { HbAlc } \leq 7 \%(\mathrm{n}=2349) \\
(\text { Labeled }=222) \\
\text { ARR }[95 \% \text { CI] }\end{array}$ & $\begin{array}{c}\text { HbAlc }>7 \%(\mathrm{n}=3723) \\
(\text { Labeled = 537) } \\
\text { ARR }[95 \% \mathrm{CI}]\end{array}$ & $\begin{array}{c}\text { HbAlc } \leq 9 \%(\mathrm{n}=4546) \\
(\text { Labeled = 479) } \\
\text { ARR }[95 \% \mathrm{CI}]\end{array}$ & $\begin{array}{c}\text { HbAlc }>9 \%(\mathrm{n}=1526) \\
\text { (Labeled = 280) } \\
\text { ARR }[95 \% \text { CI] }\end{array}$ \\
\hline White & ref & ref & ref & ref & ref \\
\hline Black & $2.38[1.93,3.02]$ & $2.76[1.85,4.17]$ & $2.08[1.63,2.74]$ & $2.41[1.78,3.14]$ & $1.82[1.25,2.54]$ \\
\hline
\end{tabular}

ARR, adjusted risk ratio; HbA1c, hemoglobin A1c.

Appendix Table 4. Sample Characteristics by Care Site*

\begin{tabular}{|c|c|c|c|}
\hline & $\begin{array}{c}\text { Primary Care } \\
(\mathrm{n}=2657) \\
\text { Mean }(S D)\end{array}$ & $\begin{array}{c}\text { Specialty Care } \\
(\mathrm{n}=3415) \\
\text { Mean }(S D)\end{array}$ & $P$ Value \\
\hline Age, years & $54.0(10.0)$ & $55.1(10.1)$ & $4.7 \times 10^{-6}$ \\
\hline BMI, $\mathrm{kg} / \mathrm{m}^{2}$ & $34.1(8.4)$ & $33.1(8.0)$ & $1.9 \times 10^{-6}$ \\
\hline \multirow[t]{2}{*}{ HbA1c, \% } & $8.0(2.0)$ & $8.1(1.9)$ & $9.9 \times 10^{-8}$ \\
\hline & $n(\%)$ & $n(\%)$ & \\
\hline \multicolumn{4}{|l|}{ Biological sex } \\
\hline Male & $1185(44.6 \%)$ & $1724(50.4 \%)$ & ref \\
\hline Female & $1472(55.4 \%)$ & $1691(49.6 \%)$ & $6.0 \times 10^{-6}$ \\
\hline \multicolumn{4}{|l|}{ Race } \\
\hline White & $401(15.1 \%)$ & 1635 (47.9\%) & ref \\
\hline Black & $2173(81.8 \%)$ & $1634(47.8 \%)$ & $2.2 \times 10^{-6}$ \\
\hline Asian & $83(3.1 \%)$ & $146(4.3 \%)$ & $1.1 \times 10^{-8}$ \\
\hline \multicolumn{4}{|l|}{ Ethnicity } \\
\hline Non-Hispanic & $2599(97.8 \%)$ & 3307 (96.8\%) & ref \\
\hline Hispanic & $58(2.2 \%)$ & $108(3.2 \%)$ & 0.025 \\
\hline \multicolumn{4}{|l|}{ Insurance status } \\
\hline Private & $1557(58.6 \%)$ & $1979(58.0 \%)$ & ref \\
\hline Medicaid & $576(21.7 \%)$ & $506(14.8 \%)$ & $1.3 \times 10^{-7}$ \\
\hline Medicare & $524(19.7 \%)$ & $930(27.2 \%)$ & $2.3 \times 10^{-7}$ \\
\hline
\end{tabular}

BMI, body mass index; HbA1c, hemoglobin A1c.

${ }^{*} P$ values for the continuous variables were calculated using the nonparametric Wilcox test. $P$ values for the categorical variables were calculated using the chi square test; in case of more than 2 categories, 1 category is shown as reference (eg, "White"). 


\begin{tabular}{|c|c|c|}
\hline & $\begin{array}{c}\text { Group 1: Primary Care }(\mathrm{n}=2657) \\
(\text { Labeled }=376) \\
\text { ARR }[95 \% \mathrm{CI}]\end{array}$ & $\begin{array}{c}\text { Group 2: } \\
\text { Specialty Care }(\mathrm{n}=3415) \\
\text { (Labeled = 383) } \\
\text { ARR }[95 \% \mathrm{CI}]\end{array}$ \\
\hline \multicolumn{3}{|l|}{ Race } \\
\hline White & ref & ref \\
\hline Black & $2.65[1.71,4.29]$ & $2.19[1.68,2.86]$ \\
\hline Asian & $0.21[0.00,0.77]$ & $1.02[0.46,1.64]$ \\
\hline \multicolumn{3}{|l|}{ Ethnicity } \\
\hline Non-Hispanic & ref & ref \\
\hline Hispanic & $0.31[0.00,0.97]$ & $1.39[0.26,3.21]$ \\
\hline \multicolumn{3}{|l|}{ Biological sex } \\
\hline Male & ref & ref \\
\hline Female & $1.04[0.84,1.29]$ & $0.97[0.80,1.17]$ \\
\hline \multicolumn{3}{|l|}{ BMI } \\
\hline Normal & ref & ref \\
\hline Overweight & $0.62[0.41,0.91]$ & $0.75[0.55,1.01]$ \\
\hline Obese & $0.67[0.47,0.93]$ & $0.76[0.57,1.01]$ \\
\hline \multicolumn{3}{|l|}{ Insurance status } \\
\hline Private & ref & ref \\
\hline Medicaid & $2.13[1.61,2.73]$ & $1.50[1.16,1.93]$ \\
\hline Medicare & $1.38[1.00,1.85]$ & $1.63[1.30,2.04]$ \\
\hline
\end{tabular}

ARR, adjusted risk ratio; BMI, body mass index. 